\title{
Research on Congestion Control Algorithm Based on New Network Model and Wavelength Buffering in IONs
}

\author{
Xinxin $\mathrm{Ma}^{1}, \mathrm{Li} \mathrm{Du}^{2} *$ \\ ${ }^{1}$ Key Laboratory of Medical Image Computing of Ministry of Education, Northeastern University, Shenyang, 110819, China \\ ${ }^{2 *}$ College of Information Science and Engineering, Northeastern University, Shenyang, 110819, China
}

\begin{abstract}
This paper studied the congestion control algorithm in the IONs and put forward two innovative algorithms. Firstly, designed a wavelength conversion algorithm which based on Partial-sparse-Limited network model (for short PLWC); Secondly, presented another wavelength conversion algorithm which based on Partialsparse-Limited network model and Wavelength Buffering (for short PLWC_WB); Finally, analyzed and verified PLWC and PLWC_WB algorithm by system simulation. The simulation results shown that PLWC algorithm achieved an outstanding performance of the network improvement compared with the existing SPWC algorithm, and comparing with the PLWC algorithm the PLWC_WB algorithm can decrease the blocking probability about $\mathbf{1 3} \%$ and increase network resource utilization about $10 \%$.
\end{abstract}

Keywords- intelligent optical networks; congestion control; wavelength conversion; wavelength buffering.

\section{INTRODUCTION}

Network congestion problem is one of the important indicators to impact the network performance. Recently, most of the researches are concentrated on packet-based IP networks, but not the IONs. Therefore, the research about congestion control algorithm in IONs is of great significance. The reasons of IONs congestion include limited wavelength resources, Wavelength-Continuity Constraint (WCC) and Link Cascade Effect (LCE). Recently, there are several techniques to solve congestion control problems, such as routing and wavelength assignment, wavelength conversion and preemption.

The paper is organized as follows. In the next section, proposed the PLWC algorithm to place wavelength converter optimally. In Section 3, the PLWC_WB algorithm is designed to solve the time imbalance problem of network. In Section 4, the simulation is made to illustrate the efficiency of the algorithm. Finally, we conclude our paper in section 5 .

\section{II.PLWC ALGORITHM DESIGN}

In Fig.1, reference 1, 2, 3 are sequentially classified into three categories: a device-level, an architecture-level, and a network-level. And it also shows the Sparse-Partial (SP)[4] and Sparse-Limited (SL)[3] network model. Although wavelength conversion algorithms based on these network models show a better performance than based on the above three basic network models, they all explore only a face of the cube. All the internal points of the cube in Fig. 1 stand for the Partial-sparse-Limited (for short PSL) network model which combine all the three basic network models in a integrated manner. Thus, we expect that wavelength conversion based on Partialsparse-Limited network model (PLWC) algorithm can achieve much better performance than the existing approaches.

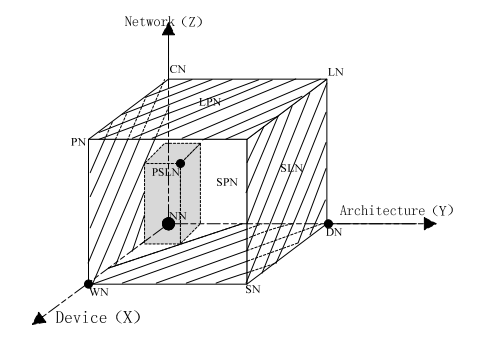

Figure 1. Conceptual view of nodes in network

The main idea of PLWC algorithm is based on PSL network model, configurating wavelength converter in the node which has the largest blocking traffic until the whole blocking probability less than the threshold $B_{w c c}$. The difficult point of PLWC algorithm is how to calculate blocking probability $B$ and the threshold $B_{w c c}$.

\section{A. Calculation of Blocking Probability}

As shown in Fig. 2, path $r$ consists of a set of ordered nodes and links, and a node can be a either wavelengthselective node (WSN) or wavelength-convertible node (WCN) depending on the capacity of wavelength conversion. Each WCN has a wavelength converter pool (WCP) which consists of $n$ wavelength converters whose conversion range is $d$.

The blocking probability $B_{W C C}$ is given by Formula (1). $\alpha$ is defined as 0.9 .

$$
B_{W C C}=\left(B_{F W C}\right)^{a}\left(B_{N W C}\right)^{1-a} \quad a \in[0,1]
$$

Where, $B_{F W C}$ and $B_{N W C}$ are the blocking probability of network after all nodes configurate WC and not configurate WC separately.

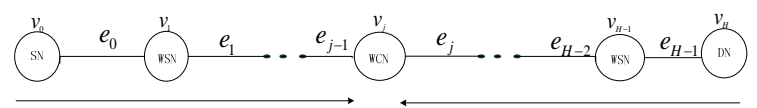

Figure 2. An H-hop path model and the corresponding notations 
The network-wide blocking probability can be represented in terms of the end-to-end blocking probabilities, as shown in Formula (2). And $a_{r}$ is the traffic load, $B_{r}$ is the blocking probability of path $r$ and $R$ is the total number of requests.

When $a_{r}$ is given, in order to obtain $B, B_{r}$ should be calculated firstly. $B_{r}$ can be divided into two parts: the blocking probability of nodes and links.

$$
B=\sum_{r \in R} \alpha_{r} B_{r} / \sum_{r \in R} \alpha_{r}
$$

(1) Blocking Probability of Multi-wavelength Link

The amount of reduction in the offered load contributed by path $r$ is given by $\alpha_{r}\left(1-B_{r \mid X_{j}=m}\right)$. Aggregating all contributions of such paths, $a_{j}(m)$ be defined as Formula (3).

And $B_{r \mid X_{j}=m}$ Conditional blocking probability of path $r$, given that the number of free wavelengths on link $e_{j}$ is equal to $m$.

$$
\alpha_{j}(m)=\sum_{\forall r: e_{j} \in \xi_{r}} a_{r}\left(1-B_{r \mid X_{j}=m}\right)
$$

Given $a_{j}(m)$, the free wavelength distribution on link $j$, is given by Formula (4).

$$
\begin{gathered}
P_{j}(m)=\frac{W(W-1) \ldots(W-m+1)}{a_{j}(1) a_{j}(2) \ldots a_{j}(m)} P_{j}(0) \\
P_{j}(0)=\left[1+\sum_{m=1}^{W} \frac{W(\mathrm{~W}-1) \ldots(\mathrm{W}-\mathrm{m}+1)}{\alpha_{j}(1) \alpha_{j}(2) \ldots \alpha_{j}(m)}\right]^{-1}
\end{gathered}
$$

(2) Blocking Probability of WCN

There are two sources of call blocking at the WCP in a WCN, they are the range blocking and the capacity blocking.

1) Model for LWC

We adopt the combinatorial LWC model to compute the conditional probability $Q(l \mid m)$ that the number of free wavelengths after wavelength conversion at WCN is equal to $l$, given that the number of free wavelengths on segment $v_{0} \rightarrow e_{j-1}$ is $m$. From the assumption of random wavelength assignment, $Q(l \mid m)$ is given by Formula (5).

$$
\begin{array}{r}
\underline{\mathrm{W}}(m)=\min (m+d, W), \overline{\mathrm{W}}(m)=\min ((2 d+1) m, W) \\
Q(l \mid m)=\frac{F(W, m, l)}{C_{W}^{m}}, \quad \underline{\mathrm{W}}(m) \leq l \leq \overline{\mathrm{W}}(m)
\end{array}
$$

We refer to reference 4 for the recursive formulations of $\Phi(W, m, l)$.

\section{2) Model for WCP}

Fig.2 is an equivalent model of WCP. $\delta_{\text {sel }}$ and $\delta_{\text {con }}$ are given by Formula (6). $\delta_{\text {sel }}$ and $\delta_{\text {con }}$ are the amount of blocked traffic in WSN and WCN. $\beta$ is the amount of range-blocked traffic of WCP, and be expressed as $\delta_{\text {sel }}-\delta_{\text {con }}$.

$$
\delta_{\text {sel }}=\sum_{\forall r: v \in V_{r, I}} \alpha_{r} B_{r \mid Y_{j=1}} \quad, \quad \delta_{c o n}=\sum_{\forall r: v \in V_{r, I}} \alpha_{r} B_{r \mid Y_{j=0}}
$$

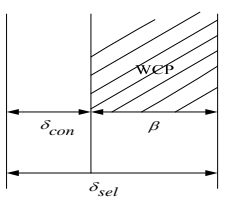

Figure 3. A model for WCP

Then, WCP can be viewed as a $M / M / n / n$ queuing system with the Poisson arrival rate $\beta$ and the unit-mean exponential service time. Therefore, the probability of capacity blocking in a WCP, denoted by $\gamma$, is given by Formula (7).

$$
\gamma=\frac{\beta^{n}}{n !}\left[1+\sum_{i=1}^{n} \frac{\beta^{i}}{i !}\right]^{-1}
$$

(3) Blocking Probability of a Multi-hop Path

1) Blocking probability of a path

The blocking probability of path $r$ can be obtained by calculating the free wavelength distribution of either $v_{0} \rightarrow e_{H}$ or $e_{0} \leftarrow v_{H}$. For the simplicity, we only present the formulations of $v_{0} \rightarrow e_{H}$.

$$
B_{r}=U_{H-1}^{-}(0)=U_{0}^{+}(0)
$$

Where, $U_{j}^{-}(m)$ and $U_{j}^{+}(m)$ are the probability of $v_{0} \rightarrow e_{j}$ and $e_{j} \leftarrow v_{H}$ has free wavelengths.

This problem can be disposed into two sub-problems: the computation of $V_{j}^{-}(k)$ in terms of $U_{j-1}^{-}(m)$, given by Formula (9).

$$
\begin{gathered}
V_{j}^{-}(k)=\sum_{m=0}^{W} U_{j-1}^{-}(m)[\gamma I(k=m)+(1-\gamma) Q(k \mid m)] \\
I(\text { cond. })= \begin{cases}1 & \text { cond } .=\text { true } \\
0 & \text { cond } .=\text { false }\end{cases}
\end{gathered}
$$

The computation of $U_{j}^{-}(f)$ in terms of $V_{j}^{-}(k)$, given by Formula (11). And $V_{j}^{-}(k)$ is probability of $v_{0} \rightarrow v_{j}$ has free wavelengths.

$$
\begin{gathered}
U_{j}^{-}(f)=\sum_{k=f}^{W} V_{j}^{-}(k) \sum_{m=f}^{W} P_{j}(m) R(f \mid k, m), \\
R(f \mid k, m)=\left(\begin{array}{c}
k \\
f
\end{array}\right)\left(\begin{array}{l}
W-k \\
m-f
\end{array}\right) /\left(\begin{array}{c}
W \\
m
\end{array}\right)
\end{gathered}
$$

\section{2) Conditional blocking probability}

By Formula (3), get to know that $\alpha_{j}(m)$ can be obtained given $B_{r \mid X_{j}=m}$. To calculate $B_{r \mid X_{j}=m}$, path $r$ be partitioned into three dependent parts: $v_{0} \rightarrow v_{j}, e_{j}$ 
and $v_{j+1} \leftarrow v_{H}$. $\mathrm{x}$ and $\mathrm{y}$ be denoted by the free wavelengths in two segments, $\mathrm{z}$ be denoted by the common free wavelengths between them. If path $r$ is blocked, there should be no common free wavelengths in the three parts. Therefore, $B_{r \mid X_{j}=m}$ can be expressed as Formula (12).

$$
B_{r \mid X_{j}=m}=\sum_{x=0}^{W} V_{j}^{-}(x) \sum_{y=0}^{W} V_{j+1}^{+}(y) \sum_{z=\max (0, x+y-W)}^{\min (x, y)} R(z \mid x, y) R(0 \mid z, m)
$$

Where, $V_{j}^{+}(k)$ is the probability of $v_{j} \leftarrow v_{H}$ has free wavelengths.

To obtain the capacity blocking probability of WCP, $B_{r \mid Y_{j}=k}$ should be calculated firstly. Path $r$ is partitioned into three segments: $v_{0} \rightarrow e_{j-1}, v_{j}$ and $e_{j} \leftarrow v_{H} . \mathrm{x}$ and $\mathrm{z}$ be denoted by the free wavelengths in two segments, $\mathrm{y}$ be denoted by the common free wavelengths between them. If path $r$ is blocked, there should be no common free wavelengths in the three parts. Therefore, $B_{r \mid Y_{j}=k}$ can be expressed as Formula (13) and (14).

$$
\begin{gathered}
B_{r \mid Y_{j}=1}=\sum_{x=0}^{W} U_{j-1}^{-}(x) \sum_{j=0}^{W} U_{j}^{+} R(0 \mid x, z) \\
B_{r \mid Y_{j}=0}=\sum_{x=0}^{W} U_{j-1}^{-}(x) \sum_{y=w-w(x)}^{-\infty(x)} Q(y \mid x) \sum_{z=0}^{W} U_{j}^{+} R(0 \mid y, z)
\end{gathered}
$$

\section{B. Wavelength Conversion Based on PSL Network Model.} follow.

The basic procedure of PLWC algorithm is listed as

Step 1: Assume LWC algorithm, find the smallest conversion range $d_{\text {min }}$ in which the blocking probability $B$ is less than or equal to $B_{W C C}$.

Step 2: Assume PSL network model, start Dijkstra and First-Fit algorithm to achieve routing and wavelength assignment.

Step 3: After all requirements have finished routing, compare the computed blocking probability $B$, if $B \leq B_{W C C}$, go to step 5, else, go to Step 4.
Step 4: Compute the amount of blocking traffics at each node. If a node is a WSN, calculate the range blocking $\delta_{\text {sel }}$, otherwise , calculate the capacity blocking $\beta \gamma$. Find the node yielding the maximum traffic blocking, and add a wavelength converter to the corresponding WCP. Go to Step 2.

Step 5: Finish placing the wavelength converter.

\section{WAVELENGTH BUFFERING SCHEME}

The main idea of wavelength buffering scheme is to set a wavelength buffer in the network, and put some wavelengths in the zone, when the whole network traffic load is low, we just start routing and wavelength assignment in the non wavelength buffer, and in the peak phase of the traffic load "release" the resources in wavelength buffer to improve the network resource utilization. We can use the following procedure to get the curve of the optimal wavelength buffer.

Step 1: Assume all the network traffics are highpriority.

Step 2: For the given network traffic load, wavelength buffer increases from zero, and increases its minimum size every time.

Step 3: When the wavelength buffer has increased to a fixed value, observe the change of blocking probability of the whole network until the fluctuation range less than $1 \%$ of its value, record the blocking probability. And continue to increase wavelength buffer. Repeat the operation of Step 3 until the emergence of the recorded minimum value of blocking probability.

Step 4: Find the smallest blocking probability in the recorded values, and the corresponding buffer capacity is the optimal wavelength buffer.

Step 5: Increase network traffic load, repeat Step 2 to Step 4 until blocking probability indicator is over the upper limit of its value. In general, this limit can be set $20 \%$. Finally, we get the optimal wavelength buffer trends with traffic load.

Table 1 is the wavelength buffer in NFSNET network topology with 128 wavelengths.

TABle I. Optimal Value Of The Wavelength Buffer

\begin{tabular}{l|l|l|l|l|l|l|l|l|l}
\hline Traffic Load (Erl) & 5 & 10 & 15 & 20 & 30 & 40 & 60 & 80 & 100 \\
\hline Value (wavelength) & 64 & 45 & 45 & 44 & 44 & 42 & 41 & 37 & 39 \\
\hline
\end{tabular}

\section{A. Starting of Wavelength Buffer}

In order to make better use of the network resource in wavelength buffer, only high-priority traffic and lowpriority traffic meeting certain conditions can start wavelength buffer. To low-priority traffic, it should meet two conditions.

i) Instantaneous traffic load of the network is greater than the setting threshold. ii) The rate of resources utilization of wavelength buffer is less than the setting threshold.

\section{B. Design of PLWC_WB}

The basic procedure of PLWC_WB algorithm is listed as follows.

Step 1: Initialize the network topology, and compute the network link state database. 
Step 2: Optimize the allocation of wavelength converters based on PSL network model.

Step 3: Calculate wavelength buffer. Then, divide the corresponding link-state database into wavelength buffer and non wavelength buffer link state database.

Step 4: Routing and wavelength assignment for the new traffic in non wavelength buffer with Dijkstra and First-Fit algorithm. If the shortest route exists and wavelength assignment success, calculate the network resource utilization of non wavelength buffer, else, go to Step 5.

Step 5: According to pre-setting level traffic priorities, for low-priority traffic, go to Step 6, for high-priority traffic, go to Step 7.

Step 6: If low-priority traffic meets with the conditions, go to Step 7, else, go to Step 8.

Step 7: Start wavelength buffer, Routing and wavelength assignment for the blocked traffic. If existing the shortest route and wavelength assignment success, calculate the network resource utilization of wavelength buffer, else go to Step 8 .

Step 8: If all business requests finish routing, algorithm ends, else go to Step 4.

\section{SIMULATION}

Fig.4, Fig.5, Fig.6 are wavelength conversion costs, blocking probability, and network improvement of wavelength conversion based on SP, SL, and PSL network model.

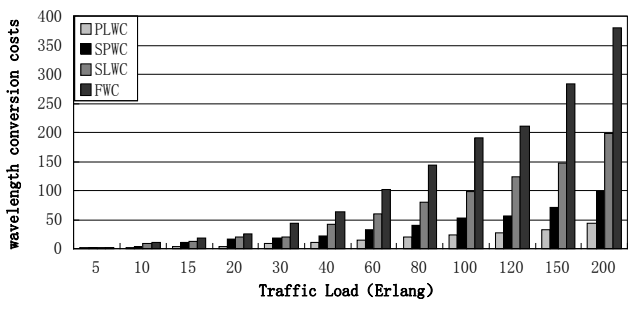

Figure 4. Wavelength conversion costs

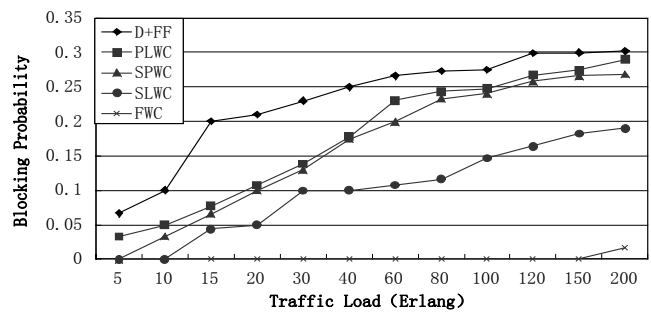

Figure 5. Network blocking probability

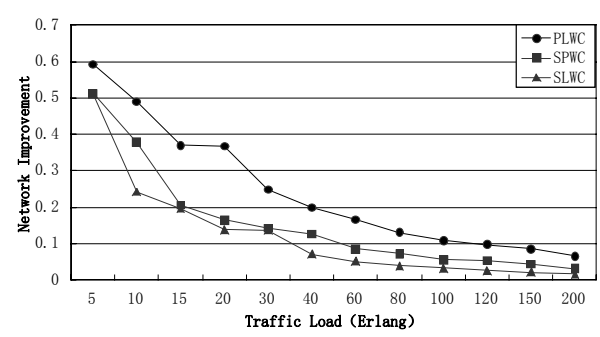

Figure 6. Network Improvement

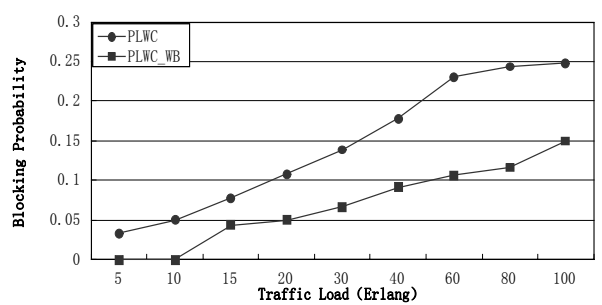

Figure 7. Network blocking probability

Fig.7, Fig.8 and Fig.9 are blocking probability, network resource utilization, network throughput, and endto-end delay of PLWC and PLWC_WB algorithm. Fig.10 is the network resource utilization of wavelength buffer and non wavelength buffer of PLWC_WB algorithm.

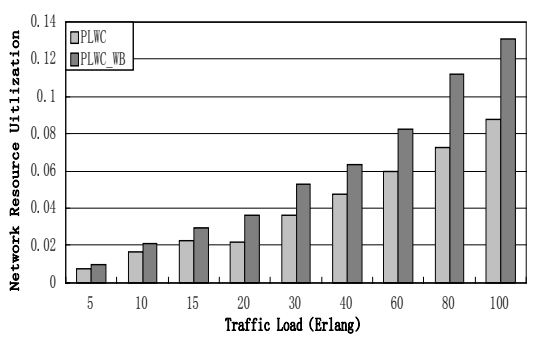

Figure 8. Network resource utilization

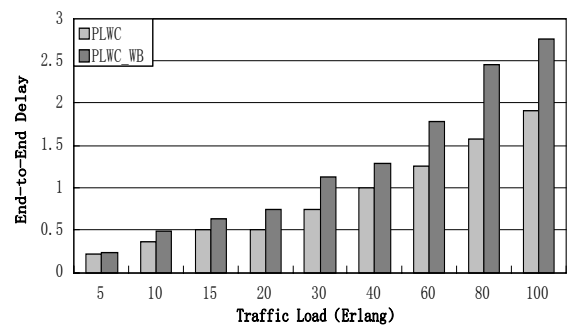

Figure 9. Network end-to-end delay 


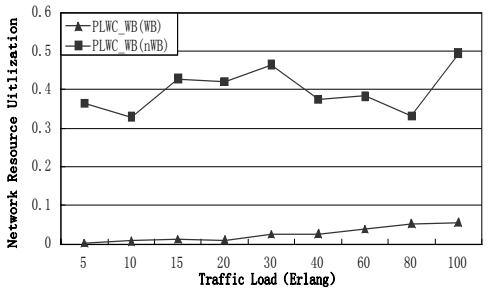

Figure 10. Network resource utilization of wavelength buffer and non wavelength buffer

\section{V.CONCLUSIONS}

This paper analyses congestion control algorithm in IONs. In order to minimize the blocking probability of the IONs, we proposed two algorithms: PLWC and PLWC_WB algorithm. PLWC algorithm solves the wavelength continuity constraint, and achieves compromise between the wavelength conversion costs and network congestion probability. The wavelength conversion costs decrease to 55 wavelengths and network improvement increases by $20 \%$ than the SPWC algorithm. On the basis of PLWC algorithm, PLWC_WB algorithm adds the WB scheme. It increases the network resource utilization by $10 \%$ and reduces the blocking probability by $13 \%$.

\section{ACKNOWLEDGEMENTS}

This research was financially supported by the Key Laboratory of Medical Image Computing of Ministry of Education.

\section{REFERENCES}

[1] D. Tanmay, K.P. Asutosh, P. Ajit. An Efficient Heuristic-based Algorithm for Wavelength Converter Placement in All-optical Networks, 2007 IEEE International Conference on Telecommunications and Malaysia International Conference on Communications,(Penang, Malaysia),2007, pp.186-190.

[2] H.Y. Jeong, S.W. Seo. A Binary (0-1) Linear Program (BLP)Formulation for the Placement of Limited-Range Wavelength Convertersin Wavelength-Routed WDM Networks. IEEE J. Lightwave Techno.,vol. 23, no. 10, Oct. 2005, pp. 30763091.

[3] X.Kang, S. Arakawa, M. Murata. An efficient algorithm for converter placement in dynamic WDM networks. 2005 IEEE International Conference on Communications, 2005. ICC 2005. 2005, pp.1884-1890

[4] X. Chu J. Liu, Z. Zhang. Analysis of Sparse-Partial Wavelength Conversion in Wavelength-Routed WDM Networks. inProc. IEEEINFOCOM’04, Apr. 2004, pp.1363-1371. 